\title{
Hemorrhage Control of Liver Injury by Short Electrical Pulses
}

\author{
Yossi Mandel ${ }^{1,2 *}$, Guy Malki ${ }^{2}$, Eid Adawi ${ }^{2}$, Elon Glassberg ${ }^{1}$, Arnon Afek ${ }^{3}$, Michael Zagetzki ${ }^{3}$, Ofer Barnea ${ }^{2}$
}

1 IDF Medical Corps, Ramat Gan, Israel, 2 Department of Bio-Medical Engineering, Tel Aviv University, Tel Aviv, Israel, 3 Sheba Medical Center, Ramat Gan, Israel

\begin{abstract}
Trauma is a leading cause of death among young individuals globally and uncontrolled hemorrhage is the leading cause of preventable death. Controlling hemorrhage from a solid organ is often very challenging in military as well as civilian setting. Recent studies demonstrated reversible vasoconstriction and irreversible thrombosis following application of microsecondslong electrical pulses. The current paper describes for the first time reduction in bleeding from the injured liver in rat and rabbit model in-vivo. We applied short $(25$ and $50 \mu \mathrm{s})$ electrical pulses of $1250 \mathrm{~V} / \mathrm{cm}$ to rats and rabbit liver following induction of standardized penetrating injury and measured the amount of bleeding into the abdominal cavity one hour post injury. We found a 60 and 36 percent reduction in blood volume in rats treated by $25 \mu$ s and $50 \mu$, respectively $(P<0.001)$. Similar results were found for the rabbit model. Finite element simulation revealed that the effect was likely nonthermal. Histological evaluation found local cellular injury with intravascular thrombosis. Further research should be done to fully explore the mechanism of action and the potential use of short electric pulses for hemorrhage control.
\end{abstract}

Citation: Mandel Y, Malki G, Adawi E, Glassberg E, Afek A, et al. (2013) Hemorrhage Control of Liver Injury by Short Electrical Pulses. PLoS ONE 8(1): e49852. doi:10.1371/journal.pone.0049852

Editor: Alexandre J. Kabla, University of Cambridge, United Kingdom

Received April 14, 2012; Accepted October 15, 2012; Published January 8, 2013

Copyright: (c) 2013 Mandel et al. This is an open-access article distributed under the terms of the Creative Commons Attribution License, which permits unrestricted use, distribution, and reproduction in any medium, provided the original author and source are credited.

Funding: This study was supported by a grant from the Israeli Ministry of defence and the IDF (Israel Defense Forces) Medical Corps. The funders had no role in study design, data collection and analysis, decision to publish, or preparation of the manuscript.

Competing Interests: The authors have declared that no competing interests exist.

*E-mail: yossi.mandel@gmail.com

\section{Introduction}

Trauma is a leading cause of death among young individuals globally. Uncontrolled non-compressible hemorrhage is the leading cause of preventable deaths $[1,2,3,4]$. Most of the battlefield hemorrhages are compressible, e.g. they can be controlled by tourniquets, haemostatic dressings or other means of direct pressure application. A recent US Army survey demonstrated increased survival rate due to early tourniquet application, while causing minimal collateral damage [5]. In contrary, controlling hemorrhage in the junctional regions (axilla, groin or neck) remains a major challenge in the battlefield and new devices are currently under evaluation [6]. Similarly, truncal hemorrhages, originating from internal cavities (such as chest, peritoneal or retroperitoneal spaces) or solid organs (e.g. liver, spleen, and kidney) are considered as non-compressible and application of effective direct external pressure is not applicable. Controlling hemorrhage from a solid organ may be challenging even in anoperation setting due to the rich vasculature and the lack of supportive connective tissue. Several techniques for controlling hemorrhage from solid organs are being studied to address this major challenge. High Intensity Focused Ultrasound (HIFU) [7,8] induces a rapid tissue temperature increase and cavitation formation, both leading to thrombosis and platelets activation [9]. This technique causes adverse reactions such as irreversible destruction of the liver and the blood vessels. Another potential treatment for liver injury is the intra-operative application of homeostatic bandages which were demonstrated to be effective in controlling liver hemorrhage in large animal models [10]. Nevertheless, these techniques, as well as others [11,12], are still under evaluation and have not yet been proven to offer a satisfactory solution to the clinical needs, especially as they all require surgical exposure of the bleeding site.

Long application of direct current has been demonstrated to cause thrombosis of a clamped blood vessel [13] [14] [15]. However, applying this technique for clinical use in hemorrhage control is not practical due to the high risk of the expected injury to the tissue. Short (sub-ms) electrical pulses have been shown to induce constriction of blood vessels and, at higher settings, thrombosis, with minimal thermal effects $[16,17,18]$. The strength-duration dependence of the thresholds of vasoconstriction and thrombosis have been recently measured for the arteries in veins in chicken embryo [19]. That study also determined that the onset time of vasoconstriction was about 10 seconds after the beginning of electrical stimulation, while thrombosis was achieved at approximately 3 minutes.

Analyzing injury data from recent conflicts, a US Army paper [20] estimated that "Effective methods for the treatment of noncompressible penetrating truncal injury will have the greatest potential to impact DOW (Died Of Wound) and KIA (Killed In Action) rates in current overseas contingency operations. The prehospital arena offers the best opportunity for impact." We believe that the technique describe in this paper has the potential to be applied in pre-hospital and hospital care for management of noncompressible hemorrhage. Our long term goal is to develop a portable device to control internal non-compressible hemorrhage from solid organs. The current paper describes for the first time that sub-millisecond electric pulses reduce the amount of bleeding in a liver injury. We also demonstrate that the temperature rise during the treatment is minimal, indicating that treatment 
mechanism is likely non-thermal. Histological analysis of treated liver further shows the thrombotic effect of electrical pulses

\section{Results}

\section{Blood Loss}

Body weight, excised liver weight and normalized excised liver weight were not significantly different between four animal groups $(\mathrm{p}>0.1)$ (Table 1). In contrast, blood loss in rats treated with $50 \mu \mathrm{s}($ EPT-50) and $25 \mu \mathrm{s}$ (EPT-25) was significantly reduced by $36 \%$ and $60 \%$, respectively, as compared to the non-treated (NT) control group ( $p<0.001$ for both groups, Table 1, Fig. 1). Blood loss in the EPT-25 group was significantly lower than in the EPT50 group $(p=0.025)$. Blood loss in the mechanical pressure (MP) group did not differ significantly from the NT group $(p=0.43)$. Similar results were found for rabbits (Table 1, Fig. 1), where blood loss in the electric pulse treatment group was reduced by more than 40 percent compared to the non-treatment group $(\mathrm{p}=0.004)$.

\section{Bio Heat Model}

Solution to the heat generation and conduction in tissue (Fig. 2) showed a mild temporal increase in liver temperature in response to 200 electric pulses. Maximal liver temperature following treatment was $39.4^{\circ} \mathrm{C}$ with $50 \mu$ s pulses and $37.9^{\circ} \mathrm{C}$ with $25 \mu \mathrm{s}$ pulses. The temperature increase was transient, and returned to normal after about 100 second following treatment. Solution to the electric problem in the rabbit electrode configuration revealed that the electrical field below the electrode was relatively uniform with maximal value of $660 \mathrm{~V} / \mathrm{cm}$ (Fig. 3). The electric field decreased along the depth of the tissue to $213 \mathrm{~V} / \mathrm{cm}$ at the lower point of the liver cut. The heat solution for the rabbit treatment showed a minimal temperature elevation of less than $0.15^{\circ} \mathrm{C}$ at the described treatment protocol.
Measurements of temperature with a thermal camera in two rabbits treated with 200 pulses of 500 Volts showed that immediately after treatment the average surface temperature below the electrodes was only mildly increased (by 0.2 and $1.04^{\circ} \mathrm{C}$ ; initial temperature were 34.3 and $35.04^{\circ} \mathrm{C}$, respectively). The mild increase in temperature is in good agreement with the bio heat solution for the rabbit case. Importantly, our bio-heat model, as well as similar published experiments (e.g. [21]) predict that maximal electrical field and temperature increase are expected to occur at the tissue surface close to the treatment electrode. The thermal camera also measures surface temperature, and therefore the small temperature increase found in liver surface temperature assess the maximum temperature rise in tissue.

\section{Histology}

We analyzed six histological specimens from 4 rats and 2 rabbits. Fig. 4 shows characteristic histological results of rat $(A, B)$ and rabbit $(\mathrm{C}, \mathrm{D})$ livers treated with short electrical pulses. Fig. $4 \mathrm{~A}$ shows a sharp demarcation line between normal and affected areas (arrows) in rat liver treated with 200 pulses of 50 microseconds with electrical field of $1250 \mathrm{~V} / \mathrm{cm}$. Higher magnification of the same preparation shows hepatocytes with eosinophilic discoloration, unclear cells borders and red blood cells clogging in large blood vessels, which might be the mechanism of hemorrhage control by electric pulses (Fig. 4 B). Rabbit liver treated with 100 pulses of 500 volts at pulse duration of $25 \mu$ s shows similar changes as in the rat liver with distinct border between treated area (left to arrows) to untreated area (Fig. $\mathbf{4} \mathbf{~ C})$. Higher magnification of the same area depicts eosinophilic cellular discoloration, picnotic nuclei in many of hepatocytes and extensive extravasation of RBC's due to clogging of blood vessels. Unaffected area looks normal at the right lower corner of the figure looks normal $($ Fig. 4 D).
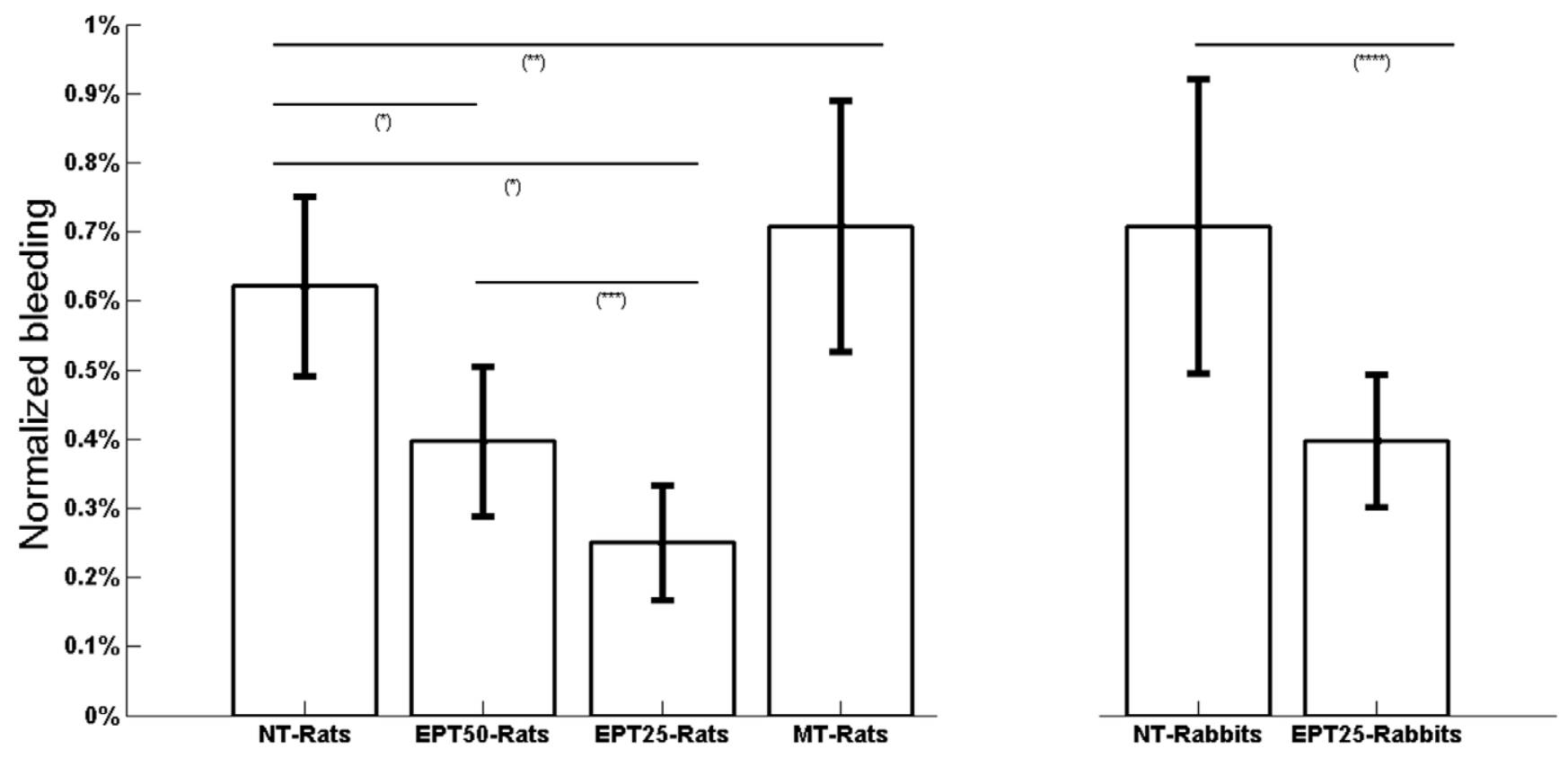

Figure 1. Experimental setup of rat (a) and rabbit (b) liver. (a) Injured rat liver (long arrows) was treated with two parallel plate electrodes (short arrows) mounted on a hand caliper adjusting for liver thickness. (b) Rabbit liver injury was treated by fix parallel plates electrodes (7.2 mm apart) (short arrows) positioned at two sides of the wound (dotted line). doi:10.1371/journal.pone.0049852.g001 
Table 1. Animal weight, excised liver weight, absolute and normalized blood loss in all animal treatment groups.

\begin{tabular}{|c|c|c|c|c|c|c|}
\hline \multirow[b]{3}{*}{$\mathbf{N}$} & \multicolumn{3}{|l|}{ Rat } & \multicolumn{3}{|l|}{ Rabbit } \\
\hline & $\begin{array}{l}\text { No treatment } \\
\text { (NT) }\end{array}$ & $\begin{array}{l}\text { Mechanical } \\
\text { Pressure (MP) }\end{array}$ & $\begin{array}{l}50 \mu \text { s pulses } \\
\text { (EPT-50) }\end{array}$ & $\begin{array}{l}25 \mu \text { s pulses } \\
\text { (EPT-25) }\end{array}$ & $\begin{array}{l}25 \mu \text { s pulses } \\
\text { (EPT-25) }\end{array}$ & No Treatment (NT) \\
\hline & 11 & 4 & 8 & 5 & 7 & 7 \\
\hline Animal weight G & $398.66 \pm 20.14$ & $408.3 \pm 49.5$ & $398 \pm 32.9$ & $392.3 \pm 33.6$ & $3196 \pm 448$ & $3106 \pm 293$ \\
\hline $\begin{array}{l}\text { Normalized Excised } \\
\text { Liver Weight } \pm \text { SD } \%\end{array}$ & $0.26 \pm 0.07$ & $0.25 \pm 0.06$ & $0.22 \pm 0.05$ & $0.21 \pm 0.05$ & NA & NA \\
\hline $\begin{array}{l}\text { Absolute blood } \\
\text { loss } \pm \text { SD G }\end{array}$ & $2.48 \pm 0.57 \pm$ & $2.83 \pm 0.65$ & $1.58 \pm 0.41$ & $0.97 \pm 0.3$ & $21.66 \pm 5.54$ & $12.63 \pm 3.31$ \\
\hline $\begin{array}{l}\text { Normalized blood } \\
\text { loss } \pm \text { SD G }\end{array}$ & $0.62 \pm 0.13$ & $0.71 \pm 0.18$ & $0.4 \pm 0.11$ & $0.25 \pm 0.08$ & $0.40 \pm 0.1$ & $0.71 \pm 0.21$ \\
\hline
\end{tabular}

doi:10.1371/journal.pone.0049852.t001

\section{Discussion}

Our results demonstrate that short electrical pulses decreased the bleeding volume from liver injury by 60 and 44 percent for rats and rabbits, respectively (Fig. 1, Table 1). The bleeding volume was not different between the NT group and the MP group in rats, suggesting that the treatment effect was not caused by the mechanical pressure applied by the electrodes per-se, but rather by the electric field applied on the tissue. Another potential explanation for the reduction of the hemorrhage could be thermal coagulation induced by increased temperature caused by Joule heating. However, thermo coagulation is usually expected at temperature of above $60-70^{\circ} \mathrm{C}[22,23]$, which according to the mathematical simulation (Fig. 2) and the experimental measurements, were not achieved even for the longer pulse duration $(50 \mu \mathrm{s})$. Further, there was no evidence for thermo coagulation in the histological sections (Fig. 4).

Another potential mechanism for hemorrhage reduction could be associated with blood vessels contraction, caused by smooth muscle activation. The liver blood flow is controlled by a pressure- flow auto regulation mechanism, which is mediated by changes in the blood vessel's muscle tonus as well as other mechanisms such as the contraction of stellate cells in response to various physiological stimuli $[24,25,26]$. Although the main branches of the hepatic and portal arteries do contain a muscular layer, it is sparely found in the more distal branches [26]. Taken together, it seems that the role of vascular contraction in the electrical induced hemorrhage control is still unclear.

We hypothesize that the hemorrhage control observed in this study is associated to endothelial layer damage, leading to thrombosis and irreversible vessel constriction. Similar effect were reported in other studies of electrochemical therapy [27]. Ramirez et al. [28] reported that electrical pulses of $850 \mathrm{~V} / \mathrm{cm}, 100$ microseconds long, caused a decrease in blood perfusion to the spleen and mesenteric arteries. The authors reported that electrical pulsing of the liver caused a decrease in perfusion, as was demonstrated by a color test. About 20 minutes after pulsing, the perfusion gradually returned to normal. Sersa et al. [29] found that 3 minutes following electric pulsing of tumors, blood flow decreased by about 80 percent and histological evaluation of the

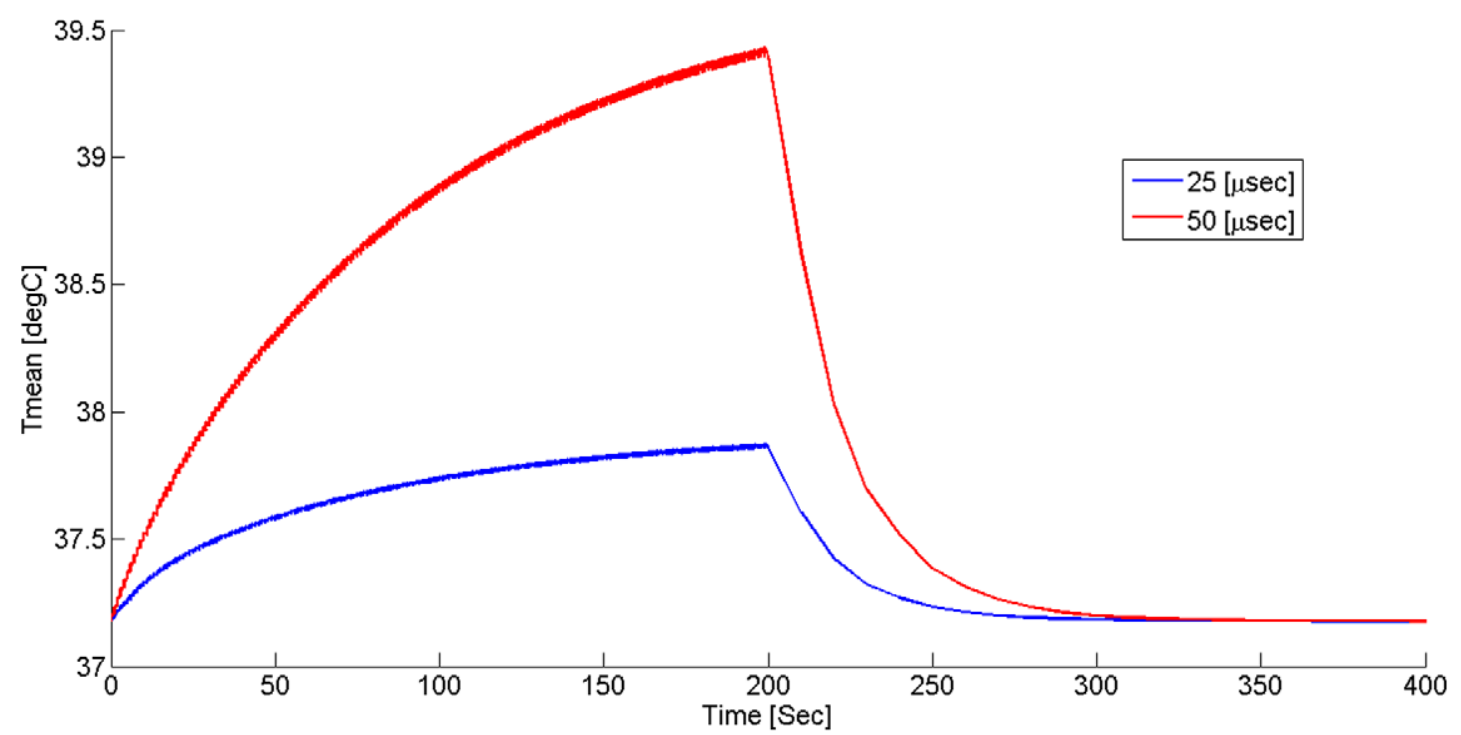

Figure 2. Normalized bleeding weight in all animal groups (rats and rabbits). Control groups were not treated. EPT50 and EPT 25 were treated by 200 pulses of 50 and $25 \mu \mathrm{s}$, respectively, in a repetition rate of $1 \mathrm{~Hz}$. Bars represents standard error. Unpaired t-test results for various comparisons are as follows: $\left({ }^{*}\right) p<0.001,\left({ }^{* *}\right) p=0.43,\left({ }^{* * *}\right) p=0.025,\left({ }^{* * * *}\right) p=0.004$.

doi:10.1371/journal.pone.0049852.g002 


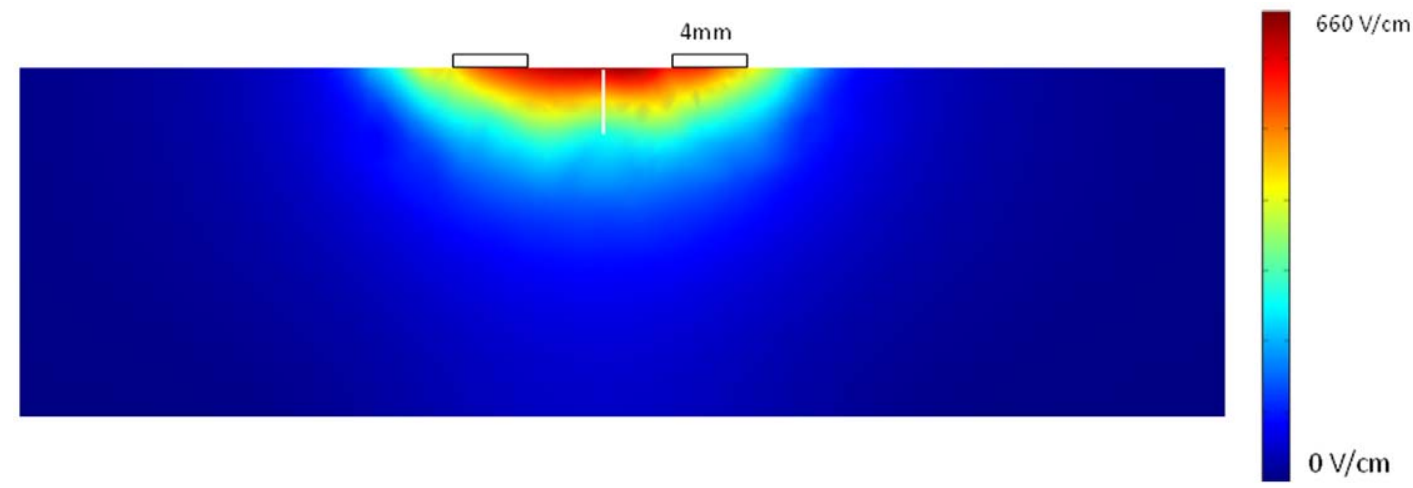

Figure 3. Calculated mean temperature versus time of rat liver treated by 200 pulses of 25 and $50 \mu$ at pulse potential of $500 \mathrm{~V}$ with repetition rate of $\mathbf{1} \mathbf{~ H z}$. Following a mild increase in temperature, there is a rapid relaxation of temperature to baseline. doi:10.1371/journal.pone.0049852.g003

endothelial cells showed that they were rounded up and swollen causing narrowing of blood vessels lumen. Eight hours after pulsing apoptosis was found in some vessels as well as extravasations and stacking of erythrocytes. The effect on blood vessels is believed to be related to immediate disruption of microfilament and microtubule cytoskeleton, liquid extravasations, increased interstitial fluid pressure and blood vessel collapse [30]. The author suggested that the effect of smooth-muscle-inducedvasoconstriction was probably less important in the case of tumor blood vessels, which have small amount of smooth muscles [27]. Palanker et al. [19] described both reversible and irreversible effects of electric pulsed on blood vessels. Histological examination revealed damage to blood vessels endothelium, which might be the reason for both reversible vasoconstriction and irreversible thrombosis. Possible cause for this endothelial damage is irreversible electroporation (IRE), which is caused by irreversible nano-size pore formation in the cellular membrane $[27,31,32]$.
The effect was probably non-thermal as the temperature rise during the treatment $\operatorname{did}$ not exceed $0.01^{\circ} \mathrm{C}$. The authors reported that the threshold voltage for reversible vasoconstriction was higher for arteries $(80 \mathrm{~V})$ than for veins $(60 \mathrm{~V})$, and did not depend on the size of the vessels. However, the threshold of thrombosis increased with the vessel diameter.

Interestingly, we found that 25 microsecond pulses were significantly more effective in reducing hemorrhage volume in rats as compared to 50 microsecond pulses (Fig. 1). Possible explanation could be associated with a local increase in liver perfusion in response to mild temperature rise in the case of 50 microsecond pulses. (Fig. 2). Previous studies showed that a local increase in liver temperature to $43^{\circ} \mathrm{C}$ caused a local increase in blood perfusion [33]. We hypothesize that such a local increase in perfusion could cause a relative increase in blood loss, partially reducing the effect of treatment. For this reasons we used $25 \mu \mathrm{s}$ pulses in the rabbit model.

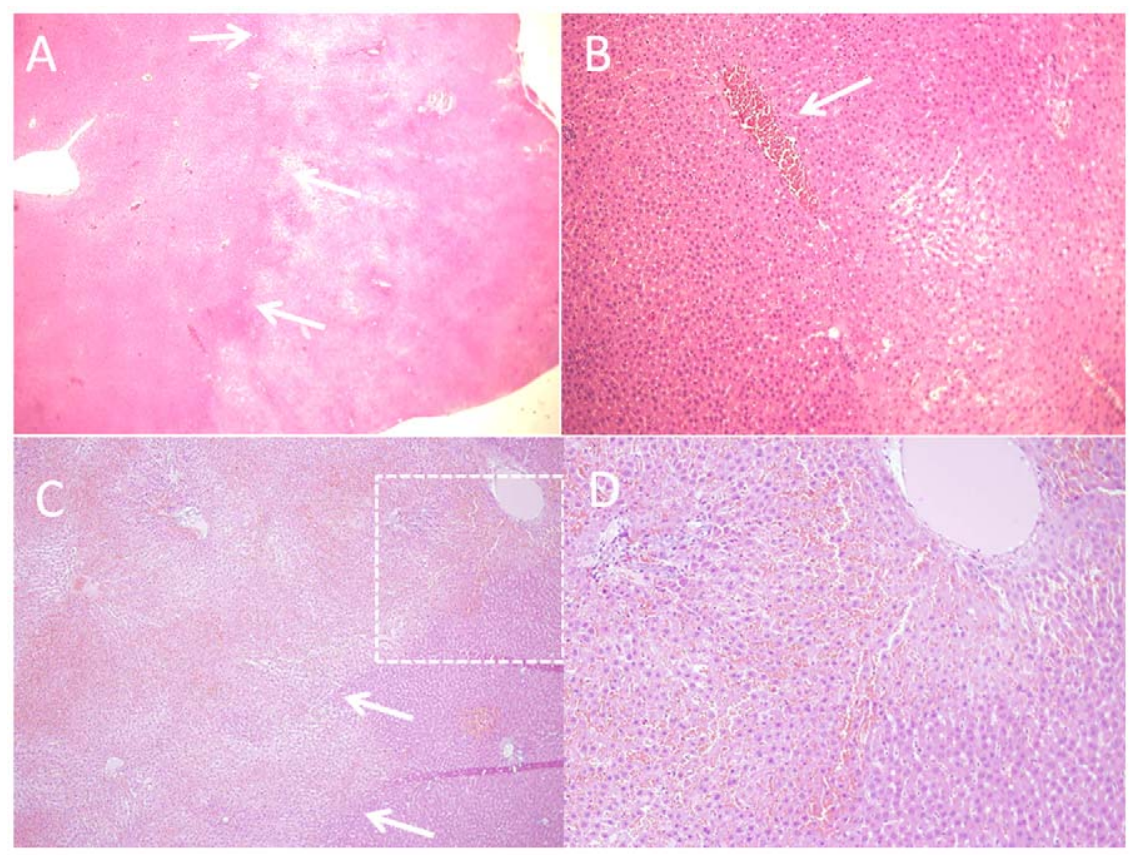

Figure 4. Simulation results of electric field around the liver cut (white line) following pulsing with $4 \mathbf{m m}$ electrodes spaced apart by $7.2 \mathrm{~mm}$.

doi:10.1371/journal.pone.0049852.g004 
In parallel to the haemostatic effect of the high electric field pulse treatment, it can cause unwanted effect on normal liver cells, as was found in histological preparation (Fig. 4). The results are similar to previously described pathological changes following irreversible electroporation in normal liver tissue [21,34], and sarcoma tumors [35]. These changes are probably caused by direct effect of electric field on cells and are not a thermal effect. The later could be caused only in instances where temperature was above $50^{\circ} \mathrm{C}$ for at least 3 minutes, which is not the case, according to our simulation. The FE simulation estimated the electric field in the area of interest in the rabbit case to be between 660 to $213 \mathrm{~V} /$ $\mathrm{cm}$ which partially overlap with irreversible electroporation thresholds. Thus, there is a potential damage effect of the short electrical pulses on the liver. However, as opposed to thermal based ablation (e.g. RF or cryo-ablation), irreversible electroporation does not induce changes in connective tissue $[36,37,38,39,40]$ enabling enhanced regenerative process of the liver [41]. Nevertheless, this potential adverse effect calls for future studies aiming an reducing the damage by changing electrode configuration or treatment protocol and parameters.

Our study has several limitations to be considered. First, blood pressure and pulse were not measured or controlled during the experiments. This could theoretically increase variance in amount of bleeding. Nevertheless, the standard variations in bleeding within the various treatment groups were low and, even without adjustment to blood pressure, were significantly different. Second, in this preliminary study we did not addressed the effect of treatment in the case of traumatic coagulopathy, which is expected in cases of severe liver trauma. Other issues to be studied in future research in larger animals are the design of electrodes in order to optimize electric field geometry, pulse parameters, better control of tissue temperature and the possible use of changes in tissue electrical properties for measuring treatment effect. Of great importance and an engineering challenge is to explore the possibility to apply the electrical field by non-invasive methods. This approach is used for few applications such as transcranial magnetic stimulation [42] or cardiac stimulation [43]. However, the electrical field induced for this application is about 2-3 orders smaller than required for the current application.

In conclusion, in this preliminary research we demonstrate that short electric pulses can significantly reduce the amount of bleeding from injured liver in a rat and rabbit model. The effect is non-thermal and possibly related to direct effect on blood vessels endothelial layer. Further research is needed in order to optimized treatment protocol and to fully expose possible potential uses for hemorrhage control in both civilian and military settings.

\section{Materials and Methods}

\section{Rats Liver Injury Protocol}

Twenty eight adult male Sprague-Dawley rats were used during the experiments. The experiment protocol was approved by the Animal Rights Council of the Israel Ministry of Health and conformed to guidelines for the humane care of animals. Rats and rabbits were supplied by Harlan Laboratories Ltd., Jerusalem, at the age of 3 months. Average animal weight is depicted in Table 1. Animals were anesthetized with an intra muscular injection of Ketamine HCI $(0.19 \mathrm{ml} / 100$ gram $)$ and Xylazine $(0.03 \mathrm{ml} / 100$ gram) solution. Following injections, the rats were placed on heating blankets and observed for full anesthesia. Additional anesthetics were given approximately every 20 minutes by titration. Twenty minutes following the administration of anesthetic drugs, a midline abdominal incision was performed and the liver was gently exposed. The median lobe of the liver was resected
$13 \mathrm{~mm}$ from lobe edge and the removed part was weighed by portable scales (Ohaus Company, model N2B110) and is referred in text as "excised liver weight". This weight was further normalized to animal total weight.

Following liver injury, rats were divided into four groups. Electrical pulse treatment group (EPT50), received 200 electrical pulses of 500 volts with pulse duration of $50 \mu$ s at pulse repetition rate of $1 \mathrm{~Hz}$. EPT25 group received 200 similar pulses given at pulse duration of $25 \mu \mathrm{s}$. The medial liver lobe of rat was placed between two customized copper electrodes attached to a commercial caliper (Fig. 5a). The distance between the 2 parallel slabs was adjustable, and determined for each animal by the liver thickness (Mean+SD electrode distance $3.98 \pm 0.56 \mathrm{~mm}$ ). A series of electrical pulses generated by Square Wave Electroporation System (ECM 830, Harvard Apparatus), operating in the monophasic mode was applied to the EPT-50 and EPT-25 groups. Thus, the mean pulsed electric field $(E=V / d)$ was about $1250 \mathrm{~V} /$ $\mathrm{cm}$.

Treatment regimen was evaluated based on preliminary experiments and literature on liver [21] and blood vessel [31] electroporation. For control we used two groups: no treatment (NT) group and mechanical pressure (MP) group, in which the electrodes were placed on the median lobe, similar to the EPT groups for 200 seconds, however, no pulses were delivered.

Following these interventions, the abdomen was closed using continuous sutures and the rats were maintained on a heated blanket for 1 hour without any further treatment. Total blood loss was measured 60 minutes after liver injury by soaking a cotton wool in the peritoneal cavity, as reported by previous authors $[44,45,46,47]$. Blood loss for each animal was normalized by its body weight. All surgical intervention and measurements were performed by the same investigator (YM).

\section{Rabbit Liver Injury Protocol}

Fourteen adult male New Zealand rabbits were used during the experiments. Rabbits were supplied by Harlan Laboratories Ltd., Jerusalem. Average animal weight is depicted in Table 1. Animals were anesthetized with an intra venous injection of Ketamine HCI $(50 \mathrm{mg} / \mathrm{kg})$ and Xylazine $(3.5 \mathrm{mg} / \mathrm{kg})$ solution followed by maintenance dosage. Twenty minutes following the administration of anesthetic drugs, a midline abdominal incision was performed, the liver was gently exposed and 3 cuts $5 \mathrm{~mm}$ deep and $3 \mathrm{~cm}$ long were performed in the three liver lobes (total 9 cuts).

Following liver injury, rabbits were divided into two groups. EPT-25 group received 200 pulses that were given with pulse duration of 25 and NT group received no treatment. In EPT-25 group rabbit liver was treated with customized electrodes comprised of two copper plates $4 \mathrm{~mm}$ wide, $29 \mathrm{~mm}$ long and spaced apart by $7.2 \mathrm{~mm}$ (Fig. 5b). Following each liver cut, the electrodes were positioned on the liver surface while the cut is in equal distances between the electrodes and a series of electrical pulses were given, similarly to the treatment described for rat liver injury.

Following these interventions, rabbit's abdomen was closed and the animals were maintained on a heated blanket for 1 hour without any further treatment. Total blood loss was measured 60 minutes after liver injury in the same method as described for rats. All surgical intervention and measurements for all experiments were performed by the same investigators (YM, GM). The pulse wave form was recorded with an oscilloscope (Tektronix TDS 220) during treatment in three rabbits. The measured waveform followed a square voltage pulse (with rise time of less than $1 \mu \mathrm{s}$ and fall time of about $1 \mu \mathrm{s}$ ) and reached the target 


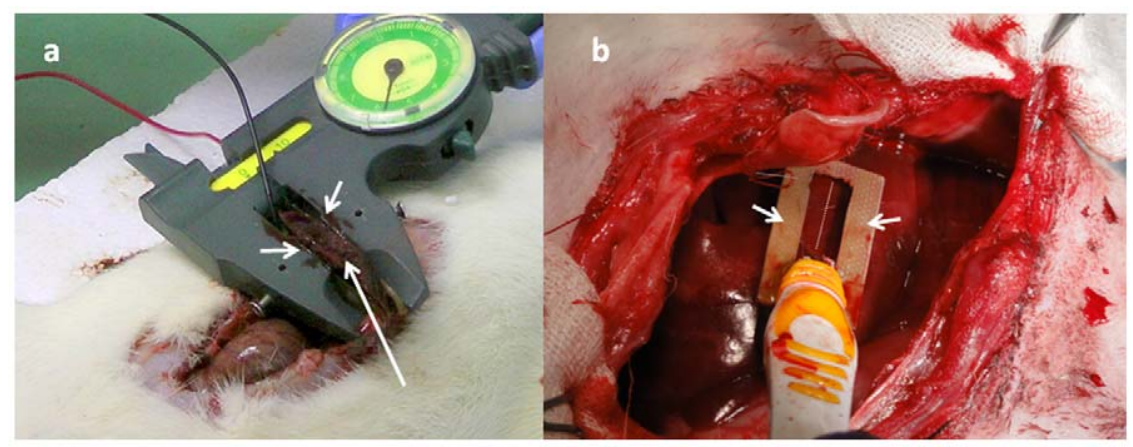

Figure 5. H\&E staining of rat $(A, B)$ and rabbit $(C, D)$ livers treated with short electrical pulses following injury. Figure $A$ shows a sharp demarcation line between normal and treated areas (arrows) in rat liver treated with 200 pulses of 50 microseconds with electrical field of $1250 \mathrm{~V} / \mathrm{cm}$. B Higher magnification of the same. C. Rabbit liver treated with 100 pulses of 500 volts at pulse duration of $25 \mu$ s shows similar changes as in A,B with distinct border between treated area (left to arrows) to untreated area. D. Higher magnification of the same area depicts eosinophilic cellular discoloration, picnotic nuclei in many of hepatocytes and extensive extravasation of RBC's due to clogging of blood vessels.

doi:10.1371/journal.pone.0049852.g005

voltage at a good accuracy (of 95-99 percent of the desired pulse potential).

\section{Finite Element Analysis and Temperature Measurement}

The thermal effects of the electrical pulses in the liver were estimated by finite element analysis of the electrical field and the resulting heat field using Comsol Multiphysics 3.5 software (COMSOL inc., Sweden). The method was previously reported by our group [48]. Rat liver was modeled as a box with dimensions of $3.5 \times 6 \times 0.5 \mathrm{~cm}$, and with $3.5 \times 0.5 \mathrm{~cm}$ rectangular electrode, simulating the real dimension of the electrodes. Rabbit liver lobes were modeled as a $2 \times 8 \times 8 \mathrm{~cm}$ box where electrodes were modeled with the real dimensions of the treatment electrodes (see Material and Methods and Fig. 4). The electric potential and field for each spatial point in the liver were calculated by the solution of Poisson's equation. Electrodes were represented as surfaces of a fixed-voltage boundary condition in which one electrode had a positive potential $\mathrm{V}_{\mathrm{p}}$ referred to as the pulse potential and the other was zero (the ground). The outer boundaries of the box were set to an insulating condition and a continuity condition was applied to all other boundaries. After solving the field (Poisson) equation, the Joule heating $(p)$ rate per unit volume $\left(\mathrm{W} / \mathrm{m}^{3}\right)$ caused by the electric field can be calculated by:

$$
p=\sigma|\nabla \phi|^{2}
$$

were $\sigma$ is tissue condictivity and $\varphi$ is the electric potential. The Joule heat generated during the electroporation treatment was then added to the Pennes bio-heat equation [49] (Eq. 2) when pulse is on and set to zero when pulse is off. The following equation was solved for different scenarios:

$$
\rho c_{p} \frac{\partial T}{\partial t}=\nabla(k \nabla T)+w_{b} c_{b}\left(T_{a}-T\right)+q^{\prime \prime \prime}+p
$$

where $k$ is the thermal conductivity of the tissue, $T$ is the temperature, $w_{b}$ is the blood perfusion, $c_{\mathrm{b}}$ is the heat capacity of the blood, $T_{a}$ is the arterial temperature, $q^{\prime \prime \prime}$ is the metabolic heat generation, $p$ is the electric heat generation, $\rho$ is the tissue density, and $c_{p}$ is the heat capacity of the tissue. The boundary conditions for the bio-heat model were divided into two subdomains, one within the body while the other was protruding out of the body cavity during treatment, exposed to the surrounding environment at $25^{\circ} \mathrm{C}$ with heat flux. The simulation calculate separately the resistive heating and temperature rise during pulse interval and the temperature relaxation during time interval between pulses. The problem was solved separately for each time interval while using the solution at the end of one time interval as the initial condition for the next one. These steps were repeated according to the number of pulses. Values used for the simulation analysis are summarized in Table 2. Following an optimization process of runtime and accuracy, body meshed was done with 2304 tetrahedral elements.

Surface temperature of two rabbit livers was measured in vivo by ThermoVisionÂ A40 infrared camera (FLIR systems, USA) immediately following pulse treatment. The camera was positioned $50 \mathrm{~cm}$ from the liver and acquired thermal pictures at autofocus mode. Thermal pictures before and after measurements were exported to Matlab $2011 \mathrm{~b}$ and the average temperature of liver surface below the electrode was calculated before and after treatment.

\section{Histology preparation}

The liver was removed immediately after euthanasia (on average 87 minutes following treatment) and fixed into formaldehyde $10 \%$. Histological slices were processed for $\mathrm{H} \& \mathrm{E}$ staining in

\begin{tabular}{|c|c|c|c|}
\hline Parameter & Symbol [units] & Value & Reference \\
\hline $\begin{array}{l}\text { Tissue Electrical } \\
\text { Conductivity }\end{array}$ & $\sigma[\mathrm{S} / \mathrm{m}]$ & 0.286 & {$[50]$} \\
\hline Thermal Conductivity & $\mathrm{k}[\mathrm{W} /(\mathrm{m} \cdot \mathrm{k})]$ & 0.512 & {$[50]$} \\
\hline Tissue Density & $\rho\left[\mathrm{Kg} / \mathrm{m}^{3}\right]$ & 1050 & {$[50]$} \\
\hline Tissue Heat Capacity & $C_{p}[J /(k g \cdot k)]$ & 3600 & {$[50]$} \\
\hline Blood Density & $\rho_{b}\left[\mathrm{Kg} / \mathrm{m}^{3}\right]$ & 1000 & {$[51]$} \\
\hline Blood Heat Capacity & $C_{b}[J /(k g \cdot k)]$ & 3640 & {$[50]$} \\
\hline Blood Perfusion Rate & $\omega_{\mathrm{b}}[1 / \mathrm{s}]$ & $1 e-3$ or $6.4 e-3$ & {$[52]$} \\
\hline Blood temperature & $\mathrm{T}_{\mathrm{b}}[\mathrm{k}]$ & 310.15 & - \\
\hline $\begin{array}{l}\text { Heat Transfer } \\
\text { Coefficient }\end{array}$ & $\mathrm{h}[\mathrm{W} /(\mathrm{m} 2 \cdot \mathrm{k})]$ & 10 & {$[53]$} \\
\hline $\begin{array}{l}\text { Surrounding } \\
\text { Temperature }\end{array}$ & $\mathrm{T}_{\mathrm{s}}[\mathrm{k}]$ & 298.15 & - \\
\hline
\end{tabular}

Table 2. Parameters used for numerical study. 
paraffin sections and then cut perpendicular to liver edge in order to demonstrate the transition between treated and untreated zones.

\section{References}

1. Kelly JF, Ritenour AE, McLaughlin DF, Bagg KA, Apodaca AN, et al. (2008) Injury severity and causes of death from Operation Iraqi Freedom and Operation Enduring Freedom: 2003-2004 versus 2006. J Trauma 64: S21-26; discussion S26-27.

2. Kauvar DS, Lefering R, Wade CE (2006) Impact of hemorrhage on trauma outcome: an overview of epidemiology, clinical presentations, and therapeutic considerations. J Trauma 60: S3-11.

3. McManus JG, Eastridge BJ, Wade CE, Holcomb JB (2007) Hemorrhage control research on today's battlefield: lessons applied. J Trauma 62: S14.

4. Scope A, Farkash U, Lynn M, Abargel A, Eldad A (2001) Mortality epidemiology in low-intensity warfare: Israel Defense Forces' experience. Injury 32: 1-3.

5. Kragh JF Jr, Littrel ML, Jones JA, Walters TJ, Baer DG, et al. (2009) Battle casualty survival with emergency tourniquet use to stop limb bleeding. J Emerg Med 41: 590-597.

6. Kragh JF Jr, Murphy C, Dubick MA, Baer DG, Johnson J, et al. (2011) New tourniquet device concepts for battlefield hemorrhage control. US Army Med Dep J: $38-48$.

7. Vaezy S, Martin R, Schmiedl U, Caps M, Taylor S, et al. (1997) Liver hemostasis using high-intensity focused ultrasound. Ultrasound Med Biol 23: 1413-1420.

8. Vaezy S, Noble ML, Keshavarzi A, Paun M, Prokop AF, et al. (2004) Liver hemostasis with high-intensity ultrasound: repair and healing. J Ultrasound Med 23: 217-225.

9. Poliachik SL, Chandler WL, Mourad PD, Ollos RJ, Crum LA (2001) Activation, aggregation and adhesion of platelets exposed to high-intensity focused ultrasound. Ultrasound Med Biol 27: 1567-1576.

10. Pusateri AE, Modrow HE, Harris RA, Holcomb JB, Hess JR, et al. (2003) Advanced hemostatic dressing development program: animal model selection criteria and results of a study of nine hemostatic dressings in a model of severe large venous hemorrhage and hepatic injury in Swine. J Trauma 55: 518-526.

11. Ahmadi-Noorbakhsh S, Azizi S, Dalir-Naghadeh B, Maham M (2012) Hyperpressure Intraperitoneal Fluid Administration for Control of Bleeding after Liver Injury. J Surg Res.

12. Velmahos GC, Spaniolas K, Duggan M, Alam HB, Tabbara M, et al. (2007) Abdominal insufflation for control of bleeding after severe splenic injury. J Trauma 63: 285-288; discussion 288-290.

13. Bourgain RH, Six F (1974) A continuous registration method in experimental arterial thrombosis in the rat. Thromb Res 4: 599-607.

14. Guarini S (1996) A highly reproducible model of arterial thrombosis in rats. J Pharmacol Toxicol Methods 35: 101-105.

15. Hladovec J (1973) The effect of some platelet aggregating and potential thrombosis-promoting substances on the development of experimental arterial thrombosis. Thromb Diath Haemorrh 29: 196-200.

16. Xin Y, Xue F, Ge B, Zhao F, Shi B, et al. (1997) Electrochemical treatment of lung cancer. Bioelectromagnetics 18: 8-13.

17. Matsushima Y, Takahashi E, Hagiwara K, Konaka C, Miura H, et al. (1994) Clinical and experimental studies of anti-tumoural effects of electrochemical therapy (ECT) alone or in combination with chemotherapy. Eur J Surg Suppl: 59-67.

18. Song Y, Li C, Li Y, Song Q Chang B, et al. (1994) Electrochemical therapy in the treatment of malignant tumours on the body surface. Eur J Surg Suppl: 4143.

19. Palanker D, Vankov A, Freyvert Y, Huie P (2008) Pulsed electrical stimulation for control of vasculature: temporary vasoconstriction and permanent thrombosis. Bioelectromagnetics 29: 100-107.

20. Blackbourne LH, Czarnik J, Mabry R, Eastridge B, Baer D, et al. (2010) Decreasing killed in action and died of wounds rates in combat wounded. J Trauma 69 Suppl 1: S1-4.

21. Edd JF, Horowitz L, Davalos RV, Mir LM, Rubinsky B (2006) In vivo results of a new focal tissue ablation technique: irreversible electroporation. IEEE Trans Biomed Eng 53: 1409-1415.

22. Graham SJ, Bronskill MJ, Henkelman RM (1998) Time and temperature dependence of MR parameters during thermal coagulation of ex vivo rabbit muscle. Magn Reson Med 39: 198-203.

23. Matsuoka C, Ishida T, Maehara T, Konishi K (2004) Numerical study of temperature distribution in tissue for thermalcoagulation therapy. Journal of Magnetism and Magnetic Materials 272-276: 2426-2427.

24. Ezzat WR, Lautt WW (1987) Hepatic arterial pressure-flow autoregulation is adenosine mediated. Am J Physiol 252: H836-845.

25. Ayuse T, Brienza N, O'Donnell CP, Robotham JL (1994) Pressure-flow analysis of portal vein and hepatic artery interactions in porcine liver. Am J Physiol 267: H1233-1242.

\section{Author Contributions}

Conceived and designed the experiments: YM GM EG OB. Performed the experiments: YM GM EG. Analyzed the data: YM GM EA AA MZ OB. Contributed reagents/materials/analysis tools: YM GM EA AA MZ. Wrote the paper: YM GM AA OB EG AA MZ.

26. Yeager VL, Anderson DJ, Taylor JJ (1985) Smooth muscle in the hepatic artery, portal vein and hepatic vein within the liver of the raccoon and guinea pig. Experientia 41: 262-265.

27. Gehl J, Skovsgaard T, Mir LM (2002) Vascular reactions to in vivo electroporation: characterization and consequences for drug and gene delivery. Biochim Biophys Acta 1569: 51-58.

28. Ramirez LH, Orlowski S, An D, Bindoula G, Dzodic R, et al. (1998) Electrochemotherapy on liver tumours in rabbits. Br J Cancer 77: 2104-2111.

29. Sersa G, Jarm T, Kotnik T, Coer A, Podkrajsek M, et al. (2008) Vascular disrupting action of electroporation and electrochemotherapy with bleomycin in murine sarcoma. Br J Cancer 98: 388-398.

30. Kanthou C, Kranjc S, Sersa G, Tozer G, Zupanic A, et al. (2006) The endothelial cytoskeleton as a target of electroporation-based therapies. Mol Cancer Ther 5: 3145-3152.

31. Maor E, Ivorra A, Mitchell JJ, Rubinsky B (2010) Vascular smooth muscle cells ablation with endovascular nonthermal irreversible electroporation. J Vasc Interv Radiol 21: 1708-1715.

32. Maor E, Ivorra A, Leor J, Rubinsky B (2007) The effect of irreversible electroporation on blood vessels. Technol Cancer Res Treat 6: 307-312.

33. Precup CG, Gonganau-Nitu D, Scurtu RR, Dindelegan G, Biro A, et al. (2010) Assessement by laser Doppler of the peripheral tumour perfusion after radiofrequency ablation for colorectal liver mestasis-experimental study. Chirurgia (Bucur) 105: 71-76.

34. Ivorra A, Rubinsky B (2007) In vivo electrical impedance measurements during and after electroporation of rat liver. Bioelectrochemistry 70: 287-295.

35. Al-Sakere B, Andre F, Bernat C, Connault E, Opolon P, et al. (2007) Tumor ablation with irreversible electroporation. PLoS One 2: el135.

36. Rubinsky B, Onik G, Mikus P (2007) Irreversible electroporation: a new ablation modality-clinical implications. Technol Cancer Res Treat 6: 37-48.

37. Onik G, Mikus P, Rubinsky B (2007) Irreversible electroporation: implications for prostate ablation. Technol Cancer Res Treat 6: 295-300.

38. Rubinsky J, Onik G, Mikus P, Rubinsky B (2008) Optimal parameters for the destruction of prostate cancer using irreversible electroporation. J Urol 180: 2668-2674.

39. Ivorra A, Al-Sakere B, Rubinsky B, Mir LM (2009) In vivo electrical conductivity measurements during and after tumor electroporation: conductivity changes reflect the treatment outcome. Phys Med Biol 54: 5949-5963.

40. Ivorra A, Rubinsky B (2006) Impedance analyzer for in vivo electroporation studies. Conf Proc IEEE Eng Med Biol Soc 1: 5056-5059.

41. Rubinsky B (2007) Irreversible electroporation in medicine. Technol Cancer Res Treat 6: 255-260.

42. Rossini PM, Rossi S (2007) Transcranial magnetic stimulation: diagnostic, therapeutic, and research potential. Neurology 68: 484-488.

43. Mouchawar GA, Bourland JD, Nyenhuis JA, Geddes LA, Foster KS, et al. (1992) Closed-chest cardiac stimulation with a pulsed magnetic field. Med Biol Eng Comput 30: 162-168.

44. Holcomb JB, McClain JM, Pusateri AE, Beall D, Macaitis JM, et al. (2000) Fibrin sealant foam sprayed directly on liver injuries decreases blood loss in resuscitated rats. J Trauma 49: 246-250.

45. Matsuoka T, Wisner DH (1996) Resuscitation of uncontrolled liver hemorrhage: effects on bleeding, oxygen delivery, and oxygen consumption. J Trauma 41: 439-445.

46. Matsuoka T, Hildreth J, Wisner DH (1996) Uncontrolled hemorrhage from parenchymal injury: is resuscitation helpful? J Trauma 40: 915-921; discussion 921-912.

47. Matsuoka T, Hildreth J, Wisner DH (1995) Liver injury as a model of uncontrolled hemorrhagic shock: resuscitation with different hypertonic regimens. J Trauma 39: 674-680.

48. Mandel Y, Rubinsky B Treatment of Uveal Melanoma by Non-Thermal Irreversible Electroporation: electrical and bio heat finite element model of the human eye. Journal Heat Transfer In Press.

49. Pennes HH (1948) Analysis of tissue and arterial blood temperatures in the resting human forearm. J Appl Physiol 1: 93-122.

50. Davalos RV, Mir IL, Rubinsky B (2005) Tissue ablation with irreversible electroporation. Ann Biomed Eng 33: 223-231.

51. Phillips M, Maor E, Rubinsky B (2011) Principles of Tissue Engineering With Nonthermal Irreversible Electroporation. Journal of Heat Transfer 133: 011004.

52. Haemmerich D, Tungjitkusolmun S, Staelin ST, Lee FT, Jr., Mahvi DM, et al. (2002) Finite-element analysis of hepatic multiple probe radio-frequency ablation. IEEE Trans Biomed Eng 49: 836-842.

53. Tangwongsan C (2003) Measurement of in vivo Endocardial and Hepatic Convective Heat Transfer Coefficient. WISCONSIN-MADISON: UNIVERSITY OF WISCONSIN-MADISON. 